\title{
Determinação do 5-hidroximetilfurfural em méis utilizando cromatografia eletrocinética capilar micelar
}

\author{
Determination of 5-hydroxymethyl-2-furaldehyde in honey by micellar eletrokinetic capillary electrophoresis
}

Sandra Jussara Nunes da SILVA ${ }^{1 \star}$, Paula Zilles SCHUCH ${ }^{1}$, Marilene Henning VAINSTEIN ${ }^{2}$, André JABLONSKI ${ }^{1}$

\section{Resumo}

Neste trabalho foi investigado o índice de HMF (5-hidroximetilfurfural) em méis comercializados em Porto Alegre - RS, utilizando Cromatografia Capilar Eletrocinética Micelar. O HMF, produto da condensação da frutose, é um indicador da qualidade e conservação do mel. Foram analisadas 11 marcas de méis comercializados na cidade de Porto Alegre. O composto estudado esteve presente em todas as amostras, em um intervalo de concentração de 0,191 a 6,206 mg. $\mathrm{kg}^{-1}$. Para quantificar o HMF presente nos méis, utilizou-se a técnica de adição de padrão. A taxa de recuperação foi de $98 \%$ e o limite de detecção foi de $0,025 \mathrm{mg} \cdot \mathrm{kg}^{-1}$. O limite permitido de HMF em méis, segundo a legislação brasileira, é de $60 \mathrm{mg} \cdot \mathrm{kg}^{-1}$.

Palavras-chave: eletroforese capilar; HMF; mel.

\begin{abstract}
In this work, the occurrence of HMF (5-hydroxymethylfurfural) in honey marketed in Porto Alegre - RS was investigated using Micellar Electrokinetic Capillary Chromatography. The HMF, which is the product of the fructose condensation, is an indicator of honey quality and conservation. Eleven types of honey commercialized in Porto Alegre were analyzed, and all of them contained HMF in a range from 0.191 to $6.206 \mathrm{mg} \cdot \mathrm{kg}^{-1}$. In order to quantify the HMF present in the samples, the technique of standard addition was employed. The recovery was $98 \%$ and the detection limit was $0.025 \mathrm{mg} \cdot \mathrm{kg}^{-1}$. The allowed limit of HMF in honey, according to the Brazilian Legislation, is $60 \mathrm{mg} . \mathrm{kg}^{-1}$. Keywords: capillar eletrophoresis; honey; HMF.
\end{abstract}

\section{Introdução}

O mel é uma substância produzida pelas abelhas a partir do néctar de flores (mel floral), de secreções de plantas ou de excreções de insetos sugadores de partes vivas das plantas (mel de melato), que são coletadas e transformadas através da evaporação da água e da adição de enzimas (CAMPOS et al., 2003; MARCHINI; MORETI; OTSUK, 2005; ROSSI et al., 1999; SILVA; QUEIROZ; FIGUEIRÊDO, 2004).

O mel é composto, na sua maior parte, de água e carboidratos - principalmente frutose e glicose - além de minerais (cálcio, cobre, ferro, magnésio, fósforo, potássio, entre outros), proteínas, aminoácidos, vitaminas, flavonóides, pigmentos e um grande número de ácidos orgânicos (BELITZ; GROSCH, 1992; DOWNEY et al., 2005; FALLICO et al., 2004; FENNEMA, 1993; FINOLA; LASAGNO; MARIOLI, 2007; LANARA, 1981; VILHENA; ALMEIDA-MURADIAN, 1999).

Além de ser apreciado por seu sabor característico e seu considerável valor nutritivo (MENDES, 1998; ROSSI, 1999), o homem tem utilizado o mel como medicamento, devido às suas propriedades antimicrobianas e anti-sépticas, e como conservante de frutas e grãos (CORTOPASSI-LAURINO; GELLI, 1991).

As diversas variedades de mel podem apresentar características distintas em função das plantas, de onde é extraído o néctar (origem botânica), localização geográfica, condições climáticas, presença de insetos sugadores e tipos de abelhas produtoras. Por esta razão, podem apresentar consistência, cor, flavor e aroma diferentes (BASTOS et al., 2002; CAMPOS et al., 2003; CAMPOS et al., 2000; DOWNEY et al., 2005; MENDES et al., 1998). Tipos comuns de plantas usadas para produção de mel são eucaliptos, citros e flores silvestres (KOMATSU; MARCHINI; MORETI, 2002; ROSSI, 1999). O mel de origem floral pode ser produzido a partir do néctar de uma única espécie vegetal - chamado monofloral - ou de mais de uma espécie - polifloral (BASTOS, 2002). Geralmente, um mel monofloral apresenta maior qualidade, com um flavor bem definido (FALLICO, 2004).

A formação de 5-hidroximetilfurfural (HMF) no mel, bem como em vários outros alimentos, deve-se à desidratação

Recebido para publicação em 27/3/2007

Aceito para publicação em 8/6/2007 (002424)

${ }^{1}$ Laboratório de Geoquímica Ambiental, Departamento de Engenharia de Minas, Escola de Engenharia, Universidade Federal do Rio Grande do Sul - UFRGS,

Av. Bento Gonçalves, 9500, prédio 75, laboratório 107, CEP 91501-970, Porto Alegre - RS, Brasil, E-mail: sandra.jussara@cnpq.pesquisador.br

${ }^{2}$ Centro de Biotecnologia, Laboratório de Fungos de Importância Médica e Biotecnológica, Universidade Federal do Rio Grande Sul - UFRGS,

Av. Bento Gonçalves, 9500, prédio 43431, laboratório 213, CEP 91501-970, Porto Alegre - RS, Brasil

${ }^{*}$ A quem a correspondência deve ser enviada 
das hexoses catalisada por ácidos (BELITZ; GROSCH, 1992). A presença no mel de açúcares simples e água em meio ácido fornece condições favoráveis à formação desse composto furânico (NOZAL et al., 2001). A estrutura química do HMF é mostrada na Figura 1.

O estudo do HMF em alimentos tem recebido atenção porque este composto e seus derivados, 5-clorometilfurfural e 5-sulfoximetilfurfural, têm apresentado atividade citotóxica, genotóxica, mutagênica e carcinogênica (TEIXIDÓ et al., 2006).

No mel, o HMF é um dos produtos de degradação mais comum, indicando "envelhecimento" do produto. Geralmente está ausente em méis recém-colhidos e sua concentração tende a crescer com o tempo (BASTOS et al., 2002; FINOLA; LASAGNO; MARIOLI, 2007; SPANO et al., 2006), sendo assim um importante indicador de qualidade do mel (SPANO et al., 2006; ZAPPALÀ et al., 2005). Níveis elevados de HMF podem indicar alterações provocadas por armazenamento prolongado em condições inadequadas, superaquecimento ou adulterações provocadas por adição de açúcar invertido (CANO et al., 2002; COCO et al., 1996; MARCHINI; MORETI; OTSUK, 2005; NOZAL et al., 2001; SILVA; QUEIROZ; FIGUEIRÊDO, 2004; WHITE, 1979).

O aquecimento do mel é muitas vezes necessário para reduzir a viscosidade, impedir a cristalização e fermentação do produto, ou destruir microrganismos contaminantes. Porém, este aquecimento favorece a formação de HMF (DOWNEY et al., 2005; FALLICO et al., 2004; TOSI; CIAPPINI; RÉ; LUCERO, 2002). Além do tempo e da temperatura de aquecimento, diferentes composições e diferentes valores de $\mathrm{pH}$ do mel podem levar a diferentes níveis de HMF (FALLICO et al., 2004).

O limite estabelecido pela legislação brasileira para HMF em méis é de $60 \mathrm{mg} \cdot \mathrm{kg}^{-1}$ (BRASIL, 2000). A recomendação do Codex Alimentarius prevê uma taxa máxima de $80 \mathrm{mg} \cdot \mathrm{kg}^{-1} \mathrm{de}$ HMF, para méis provenientes de países tropicais, pois nos países quentes o teor de HMF do mel tende a aumentar mais rapidamente durante o armazenamento (BOGDANOV; MARTIN; LÜLLMANN, 1997).

A maioria das análises de HMF em alimentos utiliza técnicas espectrofotométricas (WHITE, 1979; WINKLER, 1955) e cromatografia líquida com detector ultravioleta (FERRER et al., 2002; MENDES et al., 1998; VIÑAS; CAMPILLO; CÓRDOBA,

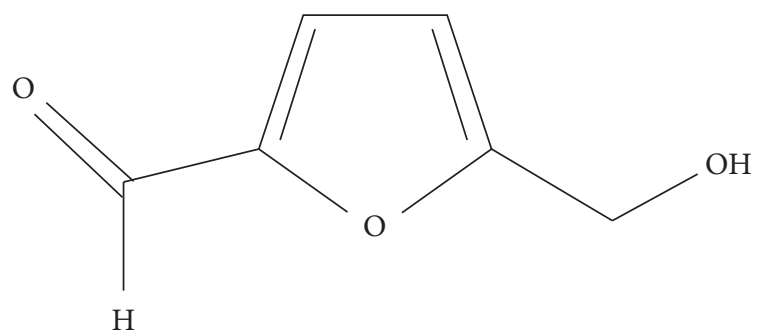

5-hidroximetil-2-furaldeido HMF

Figura 1. Estrutura química do HMF.
1992; ZAPPALÀ et al., 2005), que permite a análise simultânea de outros compostos (NOZAL et al., 2001). Em 2006, um método empregando cromatografia líquida acoplada à espectrometria de massas foi publicado (GÖKMEN; SENYUVA, 2006). Também tem sido empregada cromatografia gasosa acoplada à espectrometria de massas (HORVÁTH; MOLNÁR-PERL, 1997; TEIXIDÓ et al., 2006) para a análise de HMF em méis.

Existem três métodos oficiais recomendados pela International Honey Commission (BOGDANOV, 1999, 2007) e pela European Honey Commission (BOGDANOV; MARTIN; LÜLLMANN, 1997) para a determinação de HMF em méis: dois métodos espectrofotométricos (WHITE, 1979; WINKLER, 1955) usados em análises de rotina, e um por Cromatografia Líquida de Alta Eficiência (CLAE) (JEURING; KUPPERS, 1980). A metodologia indicada pela legislação brasileira (BRASIL, 2000) consiste na verificação do HMF utilizando-se método espectrofotométrico a 284 a $336 \mathrm{~nm}$, conforme o método 980.23 da AOAC (1998).

Dayrell e Vital (1991) analisaram amostras de méis brasileiros e encontraram valores variando de 1,10 a $248,20 \mathrm{mg} \cdot \mathrm{kg}^{-1}$. Horn et al. (1996) analisaram amostras de méis brasileiros da região nordeste e encontraram um valor médio de HMF de $113,70 \mathrm{mg} \cdot \mathrm{kg}^{-1}$. Marchini, Moreti e Otsuk (2005) analisaram amostras de méis produzidos no Estado de São Paulo e encontraram valores de HMF variando de 0,30 a 207,20 mg. $\mathrm{kg}^{-1}$. Vilhena e Almeida-Muradian (1999) analisaram 17 amostras comerciais de São Paulo. Os níveis de HMF encontrados ficaram entre 1,33 e 496,22 mg. $\mathrm{kg}^{-1}$. A amostra que apresentou maior índice de HMF - 496,22 mg.kg-1 - provavelmente estava adulterada com adição de sacarose. Silva, Queiroz e Figueiredo (2004) obtiveram teores de 0,30 a 8,96 mg. $\mathrm{kg}^{-1}$ de HMF em méis produzidos no Piauí. Souza et al. (2004) analisaram 11 amostras de méis da região semi-árida do Estado da Bahia e obtiveram valores de HMF entre 0,52 e 7,93 mg. $\mathrm{kg}^{-1}$. Sodré (2000) encontrou valores entre 1,50 a 136,00 mg.kg-1 , com média de 24,33 mg.kg-1 em amostras do litoral norte da Bahia. Alves et al. (2005) analisaram méis provenientes do município de São Gabriel, região semi-árida do Estado da Bahia, através da espectofotometria (BOGDANOV; MARTIN; LÜLLMANN, 1997) e encontraram valor médio de 5,79 mg. $\mathrm{kg}^{-1}$ e máximo de 16,54 mg. $\mathrm{kg}^{-1}$.

Nesta pesquisa, sugere-se uma técnica alternativa para a análise de HMF em méis, utilizando a cromatografia eletrocinética capilar micelar (MECC). É a primeira vez que esta técnica (MECC) é aplicada em amostras de méis para determinação de HMF. Esta técnica de separação foi descrita por Terabe et al. (1984) por modificação do método de eletroforese capilar, que até então só era aplicada para separação de solutos com carga. Esta técnica envolveu a introdução de surfactantes como o sulfato de dodecil sódio (SDS), em nível tal que micelas se formaram na solução, constituindo uma fase pseudo-estacionária.

O sistema de separação da MECC é bastante similar ao que existe em uma coluna de cromatografia líquida de partição, exceto que a fase estacionária está se deslocando ao longo da coluna a uma velocidade muito menor que a fase móvel (FOLEY, 1990). O mecanismo de separação depende da diferença de coeficientes de distribuição dos analitos entre a fase aquosa e a fase pseudo-estacionária (TERABE, 1989). A MECC apresenta 
uma série de vantagens, tais como: baixo custo do instrumental, utilização de pequeno volume de amostra, curto tempo de análise, pequeno ou nenhum consumo de solventes tóxicos ou caros, além da alta eficiência de separação. Possui alto número de pratos teóricos (100 a 200 mil) em comparação à CLAE ( 5 a 20 mil). Além disso, mudar a segunda fase em MECC é simples, pois envolve apenas a mudança da composição micelar do tampão. Na CLAE, ao contrário, a segunda fase somente pode ser alterada quando se muda o tipo de fase estacionária da coluna (SKOOG; HOLLER; NIEMAN, 2002).

\section{Material e métodos}

\subsection{Reagentes e padrões}

O padrão de 5-Hidroximetilfurfural (HMF) foi aquirido da Frig Acros. A solução estoque de $10.000 \mathrm{mg} \cdot \mathrm{kg}^{-1}$ foi preparada pela diluição de $1 \mathrm{~g}$ de HMF padrão em $100 \mathrm{~mL}$ de acetonitrila (Carlo Erba Reagentes). Para a posterior adição de padrão, foi preparada uma solução intermediária de $1000 \mathrm{mg} \cdot \mathrm{kg}^{-1}$ de HMF em acetonitrila, de onde obtivemos a solução de trabalho de $1 \mathrm{mg} \cdot \mathrm{kg}^{-1}$ de HMF.

Para o tampão, foram utilizadas soluções de SDS (dodecil sulfato de sódio) e fosfato. A solução de SDS 0,1 M (98\%) foi preparada por dissolução de $0,571 \mathrm{~g}$ de SDS $\left(\mathrm{C}_{12} \mathrm{H}_{25} \mathrm{NaO}_{4} \mathrm{~S}\right.$ SDS MW 288,38 g.mol-1 J. T. Baker) em $20 \mathrm{~mL}$ de água ultrapura (Milli-Q) fervida. A solução de hidrogeno fosfato de sódio foi preparada através de uma titulação potenciométrica do ácido fosfórico 0,033 M ( $\mathrm{H}_{3} \mathrm{PO}_{4} \mathrm{MW} 98 \mathrm{~g} \cdot \mathrm{mol}^{-1}$, Hewlett Packard) com hidróxido de sódio $0,1 \mathrm{M}$ ( NaOH MW 40 g. $\mathrm{mol}^{-1}$, Hewlett Packard). Na seqüência, o fosfato foi dissolvido com o SDS preparado, resultando num tampão SDS: fosfato $100 \mathrm{mM}$ : $33 \mathrm{mM}$.

\subsection{Obtenção e preparação das amostras}

As 11 amostras de méis de flores de eucalipto - todas inspecionadas pelo Serviço de Inspeção Federal (SIF) - foram obtidas aleatoriamente de estabelecimentos comercias de Porto Alegre. Na etapa de preparação da amostra, foram pesados $5 \mathrm{~g}$ de cada amostra e diluídos em balões volumétricos de $25 \mathrm{~mL}$ com água ultrapura (Milli-Q). Posteriormente, alíquotas das amostras foram filtradas em filtros $45 \mu \mathrm{m}$ e injetadas diretamente no equipamento.

\subsection{Equipamento e procedimentos}

As análises foram realizadas usando-se o instrumento de eletroforese capilar ${ }^{3 \mathrm{D}}$ (Hewlett-Packard-Strasse 8, D-76337, Waldbronn, Germany) equipado com um detector ultravioleta. Foi utilizado um capilar revestido com sílica fundida de diâmetro de $75 \mu \mathrm{m}$ e $55 \mathrm{~cm}$ de comprimento $(46,5 \mathrm{~cm}$ do amostrador ao detector).

Capilares novos foram condicionados inicialmente passando fluxo de $\mathrm{NaOH} 1 \mathrm{M}$ por 60 minutos a 100 mbar, seguido de fluxo de lavagem com água durante 10 minutos. Similarmente, a cada corrida o capilar foi condicionado com um flush de 1 minuto de $\mathrm{NaOH}$ 0,1 M, um flush de 1 minuto de água e, finalmente, um flush de 1 minuto com a solução tampão. Para o pós-condicionamento, foi realizado um flush de 1 minuto novamente com tampão.

A melhor separação do HMF ocorreu com uma voltagem constante de $9 \mathrm{kV}$, numa temperatura constante de $25^{\circ} \mathrm{C}$, com SDS: fosfato numa proporção de $100 \mathrm{mM}: 33 \mathrm{mM}$, observandose uma excelente resolução dos picos. $\mathrm{O}$ composto foi analisado em um comprimento de onda na região ultravioleta a $280 \mathrm{~nm}$. Foi utilizado o modo pressurizado de injeção, com uma pressão de 50 mbar por 8 segundos. O tempo total de corrida foi de 6 minutos.

\subsection{Procedimento de análise quantitativa}

A diversidade de substâncias com funções químicas variadas que compõem o mel faz com que sua matriz seja extremamente complexa. Essa condição, aliada ao fato de essa técnica não apresentar uma prévia preparação das amostras, impõe o uso de um método mais eficiente do que uma curva de calibração para a quantificação do HMF. O método escolhido, então, foi o da adição de padrão (LANÇAS, 2004; SKOOG; HOLLER; NIEMAN, 2002), o qual elimina os efeitos interferentes da matriz na quantificação do composto de interesse. Este método consiste em determinar a concentração do composto a partir de um gráfico de calibração elaborado por meio da adição sucessiva de quantidades combinadas de padrão e amostra (a concentração da solução padrão de HMF foi de $1 \mathrm{mg} \cdot \mathrm{kg}^{-1}$ ). Foram prepararadas seis combinações diferentes de soluções (padrão + amostra), de acordo com a Tabela 1. Por extrapolação do gráfico sinal $\left(\mathrm{mAU}{ }^{\star} \mathrm{s}\right)$ versus concentração de padrão $\left(\mathrm{mg} \cdot \mathrm{kg}^{-1}\right)$, obtém-se a concentração de HMF na amostra.

\section{Resultados e discussão}

\subsection{Eficiência do método}

Para a preparação da curva de cada amostra de mel, foram realizadas seis corridas de cromatografia eletrocinética capilar micelar. As curvas de calibração com adição de padrão para as amostras apresentaram uma boa linearidade na faixa de concentração entre 0,025 e $0,875 \mathrm{mg} \cdot \mathrm{kg}^{-1}\left(\mathrm{r}^{2}=0,999\right)$. A concentração de HMF para cada amostra foi calculada pela extrapolação do gráfico área do pico versus a concentração de padrão.

As análises foram realizadas em triplicata, e a média das concentrações de HMF em cada amostra estão expostas na Tabela 2. Nesta tabela também se encontram os tempos médios de migração, em minutos, do HMF em cada amostra.

Tabela 1. Volumes de padrão de HMF (1 mg. $\left.\mathrm{kg}^{-1}\right)$ e de amostra na adição de padrão.

\begin{tabular}{|c|c|c|}
\hline Solução & $\begin{array}{c}\text { Volume de padrão HMF } \\
1 \mathrm{mg} \cdot \mathrm{kg}^{-1}(\mu \mathrm{L})\end{array}$ & $\begin{array}{c}\text { Volume de } \\
\text { amostra }(\mu \mathrm{L})\end{array}$ \\
\hline 1 & 10 & 390 \\
\hline 2 & 50 & 350 \\
\hline 3 & 100 & 300 \\
\hline 4 & 200 & 200 \\
\hline 5 & 300 & 100 \\
\hline 6 & 350 & 50 \\
\hline
\end{tabular}


Tabela 2. Concentração de HMF, em mg.kg-1, e tempo de migração, em minutos, encontrados nas amostras analisadas $(n=3)$.

\begin{tabular}{ccc}
\hline Amostra & $\begin{array}{c}\text { Concentração média de } \\
\text { HMF }\left(\mathrm{mg}^{-k^{-1}}\right)\end{array}$ & $\begin{array}{c}\text { Tempo de migração } \\
\text { médio (minutos) }\end{array}$ \\
\hline A & 0,191 & 4,429 \\
B & 1,229 & 5,013 \\
C & 0,309 & 5,528 \\
D & 0,978 & 4,212 \\
E & 6,206 & 4,247 \\
F & 0,366 & 4,332 \\
G & 0,316 & 4,273 \\
H & 2,490 & 4,203 \\
I & 0,435 & 4,409 \\
J & 0,677 & 4,213 \\
K & 1,514 & 4,293 \\
\hline
\end{tabular}

\subsection{Limite de detecção e quantificação}

O limite de detecção (LOD) foi calculado para uma taxa sinal: ruído de 3:1 para o máximo volume de injeção de amostra sem perda de resolução. O limite de detecção do HMF foi de $0,025 \mathrm{mg} \cdot \mathrm{kg}^{-1}$. O limite de quantificação (LOQ) foi calculado para uma taxa sinal: ruído de 10:1, e foi de $0.0833 \mathrm{mg} \cdot \mathrm{kg}^{-1}$.

\subsection{Recuperação}

A recuperação do HMF na faixa entre 0,025 e 0,875 mg. $\mathrm{kg}^{-1}$ foi de 98 a 102\%.

\subsection{Sensibilidade}

A sensibilidade do método apresentou-se muito boa para o HMF. A sensibilidade alcançada está de acordo com os limites de controle de qualidade, sendo que, para o HMF, o limite máximo permitido é de $60 \mathrm{mg} . \mathrm{L}^{-1}$ no Brasil.

O procedimento foi aplicado para a determinação de HMF em várias amostras de méis comerciais obtidas no comércio local. Cada amostra foi analisada em triplicata.

\subsection{Repetibilidade}

A repetibilidade do método foi obtida por triplicata do procedimento analítico completo, realizado em cada uma das 11 amostras e expressa através do desvio padrão relativo (DPR). $\mathrm{O}$ valor médio da repetibilidade do método proposto ficou abaixo de $2 \%$, na faixa de concentrações estudada.

\section{Conclusões}

Todos os méis estudados apresentaram quantidades de HMF compatíveis com os valores referenciados na literatura e se enquadram dentro dos padrões de qualidade exigidos pelo Ministério da Agricultura, para comercialização no Brasil.

O método mostrou-se eficiente na quantificação do HMF, reunindo várias vantagens que facilitam a análise do mel, a fim de determinar sua qualidade. Entre as vantagens, pode-se destacar a simplicidade do método, que não requer etapas de preparação e tratamento da amostra; o baixo custo econômico e ambiental, devido ao baixo consumo de solvente; além da alta percentagem de recuperação e eficiência de separação superior aos equipamentos comumente usados. Estas características fazem desta nova técnica um caminho alternativo de alto potencial para a determinação de HMF em méis. Apesar de ser um equipamento que não existe rotineiramente em laboratórios de análises de alimentos, é economicamente mais acessível que um cromatógrafo líquido de alta eficiência ou um cromatógrafo gasoso.

\section{Agradecimentos}

Os autores agradecem à FAPERGS (Fundação de Amparo à Pesquisa do Estado do Rio Grande do Sul) pelo suporte financeiro.

\section{Referências bibliográficas}

ALVES, R. M. O. et al. Physico-chemical characteristics of honey samples of stingless bee Melipona mandacaia Smith (Hymenoptera: Apidae). Ciência e Tecnologia de Alimentos, v. 25, n. 4, p. 644-650, 2005.

A.O.A.C. ASSOCIATION OF OFFICIAL ANALYTICAL CHEMISTS Official methods of analysis of the Association of Official Analytical Chemistry. $16^{\mathrm{a}}$ ed. Maryland, 1998.

BASTOS, D. H. M. et al. Composição de voláteis e perfil de aroma e sabor de méis de eucalipto e laranja. Ciência e Tecnologia de Alimentos, v. 22, n. 2, p. 122-129, 2002.

BELITZ, H.; GROSCH, W. Química de los alimentos. $2^{2}$ ed. Zaragoza: Editorial ACRIBIA, S.A., 1992.

BOGDANOV, S. Honey quality and international regulatory standards: review by International Honey Comission. Bee World, v. 80, n. 2, p. 61-69, 1999.

BOGDANOV, S. Harmonized Methods of the International Honey Commission. Bern, Switzerland: Swiss Bee Research Centre, 2002. Disponível em: < http://www.alp.admin.ch/ themen/00502/00555/00564/ index.html?lang=de\&download=M 3wBUQCu/8ulmKDu36WenojQ1NTTjaXZnqWfVpzLhmfhnapm mc7Zi6rZnqCkkIN0fHl+bKbXrZ2lhtTN34al3p6YrY7P1oah162ap o3X1cjYh2+hoJVn6w== >. Acesso em: 8 mar. 2007.

BOGDANOV, S.; MARTIN, P.; LÜLLMANN, C. Harmonised methods of the European Honey Commission. Apidologie, p. 1-59, 1997.

BRASIL. Ministério da Agricultura. Instrução normativa 11, de 20 de outubro de 2000. Regulamento técnico de identidade e qualidade do mel. Diário Oficial da República Federativa do Brasil, Brasília, DF, 20 out. 2000, Seção 1, p.16-17.

CAMPOS, G. et al. Classificação do mel em floral ou mel de melato. Ciência e Tecnologia de Alimentos, v. 23, n. 1, p. 1-5, 2003.

CAMPOS, G. et al. Substâncias voláteis em mel floral e mel de melato. Ciência e Tecnologia de Alimentos, v. 20, n. 1, p. 18-22, 2000

CANO, C. B. et al. Mel: fraudes e condições sanitárias. Revista do Instituto Adolfo Lutz, v. 52, p. 1-4, 1992.

COCO, F. et al. High-performance liquid chromatographic determination of 2-furaldehyde and 5-hydroxymethyl-2furaldehyde in honey. Journal of Chromatography A, v. 749, p. 95-102, 1996.

CORTOPASSI-LAURINO, M.; GELLI, D. S. Analyse pollinique, propriétes physico-chimiques et action antibactérienne des miels 
d’abbeilles africanisées Apis mellifera et de Méliponinés du Brésil. Apidologie, v. 22, n. 1, p. 61-73, 1991.

DAYRELL, I. O.; VITAL, N. C. Comparação entre dois métodos oficiais para determinação de hidroximetilfurfural (HMF) em mel brasileiro. Ciência e Tecnologia de Alimentos, v. 1, n. 1, p. 137-141, 1991.

DOWNEY, G. et al.Preliminary contribution to the characterisation of artisanal honey produced on the island of Ireland by palynological and physico-chemical data. Food Chemistry, v. 91, n. 2, p. 347-354, 2005.

FALLICO, B. et al. Effects of conditioning on HMF content in unifloral honeys. Food Chemistry, v. 85, n. 2, p. 305-313, 2004.

FENNEMA, O. Química de los alimentos. $2^{\mathrm{a}}$ ed. Zaragoza: Editorial ACRIBIA, S.A., 1993.

FERRER, E. et al. High-performance liquid chromatographic determination of furfural compounds in infant formulas: Changes during heat treatment and storage. Journal of Chromatography A, v. 947, n. 1, p. 85-95, 2002.

FINOLA, M. S.; LASAGNO, M. C.; MARIOLI, J. M. Microbiological and chemical characterization of honey from central Argentina. Food Chemistry, v. 100, n. 4, p. 1649-1653, 2007.

FOLEY, J. P. Optimization of Micellar Electrokinetic Chromatography. Analytical Chemistry, v. 62, n. 13, p. 1302, 1990.

GÖKMEN, V.; SENYUVA, H. Z. Improved Method for the Determination of Hydroxymethylfurfural in Baby Foods Using Liquid Chromatography-Mass Spectrometry. Journal of Agricultural and Food Chemistry, v. 54, n. 8, p. 2845-2849, 2006.

HORN, H. et al. Méis brasileiros: resultados de análise físico-químicas e palinológicas. In: CONGRESSO BRASILEIRO DE APICULTURA, 11, 1996, Teresina. Anais... Teresina: CBA, 1996, p. 403-429.

HORVÁTH, K.; MOLNÁR-PERL, I. Simultaneous GC-MS quantitation of o-phosphoric, aliphatic and aromatic carboxylic acids, proline, hydroxymethylfurfurol and sugars as their TMS derivatives: In honeys. Chromatographia, v. 48, n. 1-2, p. 120-126, 1997.

KOMATSU, S. S.; MARCHINI, L. C.; MORETI, A. C. C. C. Análises físico-químicas de amostras de méis de flores silvestres, de eucalipto e de laranjeira, produzidos por Apis mellifera L., 1758 (Hymenoptera, Apidae) no Estado de São Paulo. 2. Conteúdo de açúcares e de proteína. Ciência e Tecnologia de Alimentos, v. 22, n. 2, p. 143-146, 2002.

JEURING, H. J.; KUPPERS, F. J. High Performance Liquid Chromatography of Furfural and Hydroxymethylfurfural in Spirits and Honey. Journal Association of Official Analytical Chemists, v. 63, n. 6, p. 1215-1218, 1980.

LANARA. Laboratório Nacional de Referência Animal. Métodos analíticos oficiais para controle de produtos de origem animal e seus ingredientes. II - Métodos físicos e químicos. Mel. Brasília: Ministério da Agricultura, 1981. v. 2, cap. 25, p. 1-15.

LANÇAS, F. Validação de métodos cromatográficos de análise. $1^{\mathrm{a}}$ ed. São Carlos: RiMa, 2004.

MARCHINI, L. C.; MORETI, A. C. C. C.; OTSUK, I. P. Análise de agrupamento, com base na composição físico-química, de amostras de méis produzidos por Apis mellifera L. no Estado de São Paulo. Ciência e Tecnologia de Alimentos, v. 25, n. 1, p. 8-17, 2005.

MENDES, E. et al. Quality evaluation of portuguese honey. Carbohydrate Polymers, v. 37, p. 219-223, 1998.

NOZAL, M. J. et al. High-performance liquid chromatographic determination of methyl anthranilate, hydroxymethylfurfural and related compounds in honey. Journal of Chromatography A, v. 917, n. 1-2, p. 95-103, 2001.

ROSSI, N. F. et al. Análise da adulteração de méis por açúcares comerciais utilizando-se a composição isotópica de carbono. Ciência e Tecnologia de Alimentos, v. 19, n. 2, p. 199-204, 1999.

SILVA, C. L.; QUEIROZ, A. J. M.; FIGUEIRÊDO, R. M. F. Caracterização físico-química de méis produzidos no Estado do Piauí para diferentes floradas. Revista Brasileira de Engenharia Agrícola e Ambiental, v. 8, n. 2/3, p. 260-265, 2004.

SKOOG, D. A.; HOLLER, F. J.; NIEMAN, T. A. Princípios de Análise Instrumental. 5 ed. Porto Alegre: Bookmann, 2002. 836 p.

SODRÉ, G. S. Características físico-químicas e análises polínicas de amostras de méis de Apis mellifera L., 1758 (HYMENOPTERA: APIDAE) da região litoral norte do Estado da Bahia. Piracicaba, SP, 2000. 83 p. Dissertação - (Mestrado), Escola Superior de Agricultura "Luíz de Queiroz", Universidade de São Paulo.

SOUZA, B. A. et al. Características físico-químicas de amostras de mel de Melipona asilvai (Hymenoptera: Apidae). Ciência Rural, v. 34, n. 5, p. 1623-1624, 2004.

SPANO, N. et al. An RP-HPLC determination of 5-hydroxymethylfurfural in honey. The case of strawberry tree honey. Talanta, v. 68, n. 4 , p. 1390-1395, 2006.

TEIXIDÓ, E. et al. Analysis of 5-hydroxymethylfurfural in foods by gas chromatography-mass spectrometry. Journal of Chromatography A, v. 1135, n. 1, p. 85-90, 2006.

TERABE, S. Electrokinetic chromatography: An interface between electrophoresis and chromatography. TrAC Trends in Analytical Chemistry, v. 8, n. 4, p. 129-134, 1989.

TERABE, S. et al. Electrokinetic separations with micellar solutions and open-tubular capillaries. Analytical Chemistry, v. 56, n. 1, p. 111-113, 1984.

TOSI, E. et al. Honey thermal treatment effects on hydroxymethylfurfural content. Food Chemistry, v. 77, p. 71-74, 2002.

VILHENA, F; ALMEIDA-MURADIAN, L. B. Análises físico-químicas de méis de São Paulo. Mensagem Doce, v. 53, p. 17-19, 1999.

VIÑAS, P.; CAMPILLO, N.; CÓRDOBA, M. H. Simultaneous liquid chromatographic analysis of 5-(hidroxymethyl)-2-furfuraldehyde and methyl anthranilate in honey. Food Chemistry, v. 44, n. 1, p. 67-72, 1992.

WHITE Jr., J. W. Spectrophotometric Method for Hydroxymethylfurfural in Honey. Journal Association of Official Analytical Chemists, v. 62 , n. 3, p. 509-514, 1979.

WINKLER, O. Beitrag zum Nachweis und zur Bestimmung von Oxymethylfurfurol in Honig und Kunsthonig. Zeitschrift für Lebensmitteluntersuchung und -Forschung A, v. 102, n. 3, p. 161-167, 1955.

ZAPPALÀ, M. et al. Methods for the determination of HMF in honey: a comparison. Food Control, v. 16, n. 3, p. 273-277, 2005. 